\title{
Active-Site Tryptophan, the Target of Antineoplastic C-Terminal Binding Protein Inhibitors, Mediates Inhibitor Disruption of CtBP Oligomerization and Transcription Coregulatory Activities $\mathrm{s}$
}

\author{
M. Michael Dcona, Priyadarshan K. Damle, Francisco Zarate-Perez, Benjamin L. Morris,
} Zaid Nawaz, Michael J. Dennis, Xiaoyan Deng, Sudha Korwar, Sahib J. Singh, Keith C. Ellis, William E. Royer, Dipankar Bandyopadhyay, Carlos Escalante, and Steven R. Grossman

\begin{abstract}
Departments of Internal Medicine (M.M.D., P.K.D., Z.N., M.J.D., S.J.S., S.R.G.), Human and Molecular Genetics (B.L.M., S.R.G.), Physiology and Biophysics (F.Z.-P., C.E.), Medicinal Chemistry (S.K., K.C.E.), and Biostatistics (X.D., D.B.) and Massey Cancer Center (K.C.E., D.B., C.E., S.R.G.), Virginia Commonwealth University, Richmond, Virginia; and Department of Biochemistry and Molecular Pharmacology, University of Massachusetts Medical School, Worcester, Massachusetts (W.E.R.)
\end{abstract}

Received September 11, 2018; accepted April 21, 2019

\begin{abstract}
C-terminal binding proteins (CtBP1/2) are oncogenic transcriptional coregulators and dehydrogenases often overexpressed in multiple solid tumors, including breast, colon, and ovarian cancer, and associated with poor survival. CtBPs act by repressing expression of genes responsible for apoptosis (e.g., PUMA, BIK) and metastasis-associated epithelial-mesenchymal transition (e.g., CDH1), and by activating expression of genes that promote migratory and invasive properties of cancer cells (e.g., TIAM1) and genes responsible for enhanced drug resistance (e.g., MDR1). CtBP's transcriptional functions are also critically dependent on oligomerization and nucleation of transcriptional complexes. Recently, we have developed a family of CtBP dehydrogenase inhibitors, based on the parent 2-hydroxyimino3-phenylpropanoic acid (HIPP), that specifically disrupt cancer cell viability, abrogate CtBP's transcriptional function, and block polyp formation in a mouse model of intestinal polyposis
\end{abstract}

that depends on CtBP's oncogenic functions. Crystallographic analysis revealed that HIPP interacts with CtBP1/2 at a conserved active site tryptophan (W318/324; CtBP1/2) that is unique among eukaryotic D2-dehydrogenases. To better understand the mechanism of action of HIPP-class inhibitors, we investigated the contribution of W324 to CtBP2's biochemical and physiologic activities utilizing mutational analysis. Indeed, W324 was necessary for CtBP2 self-association, as shown by analytical ultracentrifugation and in vivo cross-linking. Additionally, W324 supported CtBP's association with the transcriptional corepressor CoREST, and was critical for CtBP2 induction of cell motility. Notably, the HIPP derivative 4-chloro-HIPP biochemically and biologically phenocopied mutational inactivation of CtBP2 W324. Our data support further optimization of W318/W324-interacting CtBP dehydrogenase inhibitors that are emerging as a novel class of cancer cell-specific therapeutic.

\section{Introduction}

C-terminal binding proteins (CtBP) $1 / 2$ play central roles as transcriptional coregulators in metazoan development and human diseases alike (Chinnadurai, 2000). Although a precise mechanism of coregulation is yet to be described, a plausible

S.R.G. was supported by a Virginia Commonwealth University Massey Cancer Center Pilot Grant and National Institutes of Health National Cancer Institute [Grant P30CA016059]. W.E.R. was supported by National Institutes of Health National Institute of General Medical Sciences [Grant R01GM119014].

P.K.D., F.Z.-P., and B.L.M. contributed equally to this work.

https://doi.org/10.1124/mol.118.114363.

S This article has supplemental material available at molpharm.aspetjournals.org. mechanism involves CtBP oligomerization in the presence of $\mathrm{NAD}(\mathrm{H})$, where one or more $\mathrm{CtBP}$ monomers interact with DNA-interacting proteins (e.g., ZNF516) (Li et al., 2017). Following oligomerization, chromatin modifiers can be recruited to the CtBP oligomer to form a promoter-bound supercomplex that actively regulates transcription (Shi et al., 2003).

Mounting evidence also suggests that $\mathrm{CtBP}$ plays a central role in multiple diseases, including cancer (Dcona et al., 2017). Both CtBPs act as oncogenic transcriptional coregulators and are overexpressed and genomically amplified in multiple cancer types, including breast, colon, and ovarian cancer. Elevated expression levels of CtBP are associated with worse prognosis and survival (Straza et al., 2010; Barroilhet et al., 2013). The causal effect of CtBP on cancer severity could be

ABBREVIATIONS: AUC, analytical ultracentrifugation; CD, catalytic domain; CtBP, C-terminal binding protein; DMEM, Dulbecco's modified Eagle's medium; DSG, disuccinimidyl glutarate; DTT, dithiothreitol; EV, empty-vector; FDR, false discovery rate; GST, glutathione-S-transferase epitope; HIPP, 4-chloro-2-(hydroxyimino)-3-phenylpropanoic acid; IB, immunoblotting; IP, immunoprecipitation; mt, mutant; MTOB, 4(methylthio)-2-oxobutanoic acid; NBD, nucleotide-binding domain; qPCR, quantitative polymerase chain reaction; TBS-T, Tris-buffered saline, $0.1 \%$ Tween; WB, wash buffer; wt, wild-type. 
due to CtBP hyperactivity, which has been found to modulate genes that regulate apoptosis, epithelial-mesenchymal transition, and DNA repair (Stankiewicz et al., 2013). Additionally, CtBP2 has also been described as a transforming oncoprotein that is central to the evolution of neoplasia driven by Apc mutation (Sumner et al., 2017). In light of this evidence, CtBP could be considered a potential therapeutic target in cancer. Our group has developed CtBP inhibitors targeting the dehydrogenase domain that are able to modulate CtBP target genes, induce selective cytotoxicity in cancer cells, and attenuate polyposis in the Apc $\mathrm{min} /+$ mouse model of human familial adenomatous polyposis (Straza et al., 2010; Korwar et al., 2016; Sumner et al., 2017; Chawla et al., 2018).

Chemically, CtBP dehydrogenase-domain inhibitors were designed to assimilate the structural attributes of 2-keto-4methylthiobutyrate (MTOB), an $\alpha$-ketoacid substrate of CtBP that acts as inhibitor of its dehydrogenase activity at higher concentration (Fig. 1A) (Straza et al., 2010). Additionally, the cocrystal structure of $\mathrm{CtBP} 1 / 2$ and $\mathrm{MTOB}$ revealed that MTOB's thioether functional group interacts with the indolyl ring of CtBP1/2 tryptophan residue 318/324 (denoted as W324 in this work), whereas a hydrophilic water-filled cavity links MTOB with the phosphate group of $\operatorname{NAD}(\mathrm{H})$ (Hilbert et al., 2014). This led to rational design of a more potent competitive inhibitor, hydroxyl-imino phenylpyruvate (HIPP), which included replacement of the oxo-group with a nonreducible imino-hydroxyl functional group, and replacement of the thioether group with a phenyl ring that enhanced binding efficiency of the inhibitor with W324 as a result of strong $\pi-\pi^{*}$ stacking between the phenyl and indolyl rings (Hilbert et al., 2015; Korwar et al., 2016). Notably, the W324 residue is unique to $\mathrm{CtBP}$ among all eukaryotic $\mathrm{NAD}(\mathrm{H})$-dependent $\mathrm{D} 2$ dehydrogenases and has been previously hypothesized to be critical for the formation of higher-order oligomers of CtBP (Madison et al., 2013).

In light of this evidence, it is imperative to understand the physiologic role for conserved W318/324 in CtBP1/2 activities, as this residue is the key affinity target of the parent substrate competitive inhibitor HIPP that has already exhibited promising preclinical antineoplastic activity (Korwar et al., 2016; Sumner et al., 2017; Chawla et al., 2018). To better understand the biologic effect of W318/324targeted inhibitors on CtBP function, we have used sitedirected mutagenesis, wherein W324 was mutated to simulate the conditions of inhibitor interaction/inhibition of W324 biochemical activity. Using this tool, we investigated the changes in the intrinsic activities of CtBP2, such as oligomerization, transcriptional coregulation, and induction of cell migration, correlating the effects of W318/324 mutation with the observed biophysical and biologic effects of HIPP-class inhibitors.

\section{Materials and Methods}

\section{Antibodies and Immunoblot Analyses}

Antibodies used in immunoblotting (IB) and immunoprecipitation (IP) assays are CtBP2 antibody (discontinued by SCBT; E-16: sc-5966), CtBP2 (BD Biosciences), His-probe antibody (discontinued by SCBT; H-15: sc-803), glutathione-S-transferase epitope (GST) antibody (B-14: sc-138) from SCBT, Vinculin (4650T; Cell Signaling Technology), and CoREST antibody (Bethyl Antibody, A300-130A). All antibodies were used at dilutions suggested by the manufacturers. For immunoblot analysis, indicated mass of total protein extract was boiled at $95^{\circ} \mathrm{C}$ in sample buffer, followed by separation on SDS-PAGE (Novex gels), and then transferred onto nitrocellulose membrane $(0.45-\mu \mathrm{m}$ porosity) (GE Healthcare). The membrane was incubated for 1 to 2 hours in blocking buffer [Tris-buffered saline, $0.1 \%$ Tween (TBS-T), 5\% nonfat dry milk], followed by incubation overnight at $4{ }^{\circ} \mathrm{C}$ with the primary antibody solubilized in blocking buffer with sodium azide $(0.01 \%)$. After $3 \times$ washes of 5 minutes with TBS-T, the blot was incubated with Alexa Fluor 680 or $790 \mathrm{~nm}$ secondary antibodies (Invitrogen) for 1 hour in TBS-T and visualized on a Bio-Rad imager.

\section{Cell Culture}

Cells [wild-type (wt) or CRISPR] were maintained according to the American Type Culture Collection's recommendation. HCT116; p53-/- (Straza et al., 2010), HCT116;CtBP2-/- (Chawla et al., 2018), and MDA-MB-231 cells were maintained in RPMI 1640 or Dulbecco's modified Eagle's medium (DMEM) supplemented with 10\% (v/v) FBS
A

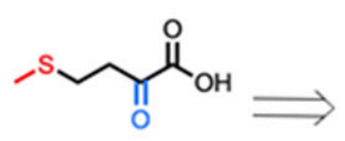

1

B Full length CtBP2 (F.L)

N-terminal Dehydrogenase domain

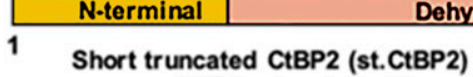

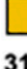

31

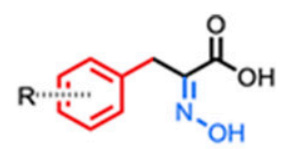

2

$\mathbf{R}=4-\mathrm{Cl}, 2-\mathrm{OMe}$

C

\begin{tabular}{|c|c|}
\hline CtBP2 mutants & Functions \\
\hline CtBP2-G189A & $\begin{array}{c}\text { Nucleotide } \\
\text { binding }\end{array}$ \\
\hline CtBP2-R272A & Catalysis \\
\hline CtBP2-H321A & Catalysis \\
\hline $\begin{array}{c}\text { CtBP2- } \\
\text { W324G/A/F }\end{array}$ & $\mathrm{TS} / \mathrm{IB}$ \\
\hline
\end{tabular}

Fig. 1. (A) MTOB (1) HIPP derivatives (2). (B) Graphical representation of full-length (F.L.) and short truncated (st.) versions of CtBP2. (C) Table listing mutants and their functions. TS/IB (Tetramer Switch/Inhibitor-binding residue). 
and penicillin-streptomycin, unless specified. Cells were maintained in a humidified incubator equilibrated with $5 \% \mathrm{CO}_{2}$ at $37^{\circ} \mathrm{C}$. All of the cells were authenticated by examination of morphology and growth characteristics and were confirmed to be mycoplasma-free using $4^{\prime}, 6^{\prime}$ diamidino-2-phenylndole staining.

\section{Site-Directed Mutagenesis and Polymerase Chain Reaction}

A pET28a vector containing the truncated, 31-364 amino-acid, histagged CtBP2 cDNA was used as a template for site-directed mutagenesis using round-the-horn amplification technique. Briefly, nonoverlapping primers amplifying in opposing directions were designed to create point mutations. Primers were phosphorylated, and polymerase chain reaction was performed, followed by DpnI digestion and overnight ligation with T4 DNA ligase. DH5 $\alpha$ chemicalcompetent cells (Invitrogen) were transformed using the ligation products and selected on LB plates supplemented with $30 \mu \mathrm{g} / \mathrm{ml}$ kanamycin. Successful mutants were confirmed by sequencing. Informative mutations, as determined by in vitro $\mathrm{NAD}(\mathrm{H})$ reaction curves, were substituted for wt codons into full-length CtBP2, carrying C-terminal V5 tag, in a modified pcDNA3.0 mammalian expression vector that also expresses GFP and puromycin acetyltransferase as a chimeric protein. Mutant CtBP2 expression plasmids included in the study incorporated the G189A, R272A, H321A, and W324G/A/F mutations. In addition, empty-vector (EV) and a plasmid-encoding wt CtBP2 were utilized (Morris, 2016).

\section{Protein Expression and Purification}

Standard culture procedures were followed, as reported in our previous work (Hilbert et al., 2015). Briefly, BL21-DE3 (RIL) cultures were induced at an optical density 600 of $0.5-0.6$ by addition of $0.5 \mathrm{mM}$ isopropyl- $\beta$-D-thiogalactopyranoside, and the temperature was reduced to $30^{\circ} \mathrm{C}$ from $37^{\circ} \mathrm{C}$. Cells were harvested 5 hours after induction, pelleted, and resuspended in $30 \mathrm{ml}$ buffer containing Complete

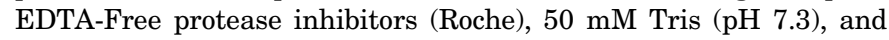
$100 \mathrm{mM} \mathrm{NaCl}$. Cells were then lysed using a high-pressure homogenizer and treated with DNase I. After pelleting, the soluble fraction was collected and incubated with $5 \mathrm{ml}$ nickel beads (Goldbio/Thermo Scientific) with gentle stirring at $4^{\circ} \mathrm{C}$ for 90 minutes. The lysate and beads were applied to a disposable column (Bio-Rad), and the lysate was allowed to flow through. Then beads were washed with $25 \mathrm{ml}$ wash buffer [WB; $50 \mathrm{mM}$ Tris $\mathrm{pH} 7.3,300 \mathrm{mM} \mathrm{NaCl}, 0.2 \mathrm{mM}$ EDTA, $1 \mathrm{mM}$ dithiothreitol (DTT), $40 \mathrm{mM}$ imidazole], followed by $50 \mathrm{ml}$ WB supplemented with $0.5 \%$ Triton-X 100, $25 \mathrm{ml} \mathrm{WB}, 50 \mathrm{ml}$ WB supplemented with $2.0 \mathrm{M} \mathrm{NaCl}$, and then $25 \mathrm{ml} \mathrm{WB}$. To remove excess cofactor [by converting $\mathrm{NAD}(\mathrm{H})$ to the weaker binding $\mathrm{NAD}^{+}$], $10 \mathrm{ml}$ WB supplemented with $250 \mathrm{mM}$ sodium pyruvate was incubated for 5 minutes on the column at $4^{\circ} \mathrm{C}$. Sodium pyruvate incubation was repeated five times. Beads were washed with a final $25 \mathrm{ml} \mathrm{WB}$ before elution with $300 \mathrm{mM}$ imidazole. Eluted protein was dialyzed into dialysis buffer (50 mM Tris, $\mathrm{pH}$ 7.3, $300 \mathrm{mM} \mathrm{NaCl}, 2 \mathrm{mM}$ DTT, $1 \mathrm{mM}$ EDTA) supplemented with $10 \%$ glycerol, flash frozen, and stored at $-80^{\circ} \mathrm{C}$ for long-term or stored at $4^{\circ} \mathrm{C}$ for usage within 1 or 2 days.

\section{Deletion of CtBP2 by CRISPR-Cas9 in MDA-MB-231 Cells}

The coordinates of the targeted sequence are 124998142-124998161 (TGCAGACGGGATGTTGCACA) in Homo sapiens chromosome 10, GRCh38.p7 Primary Assembly, Sequence ID: NC_000010.11. Annealed DNA oligonucleotides that coded for the target-specific CRISPR RNA were ligated with linear GeneArt CRISPR nuclease vector with orange fluorescence protein (catalogue number: A21174; Thermo Fisher Scientific). Electrocompetent Escherichia coli (DH1OB) were transformed overnight on $100 \mu \mathrm{g} / \mathrm{ml}$ ampicillin-supplemented LB-agar plates. The plasmid DNA was verified by sequencing. MDA-MB-231 cells at $70 \%$ confluency, grown in DMEM supplemented with $10 \%$ FBS in a $100-\mathrm{mm}$ dish, were transfected with $6 \mu \mathrm{g}$ plasmid DNA and incubated at $37^{\circ} \mathrm{C}$ $\left(5 \% \mathrm{CO}_{2}\right)$ for 72 hours. Orange fluorescence protein-positive cells were single-cell sorted in a 96-well plate using an Aria-BD FACSAria II High-Speed Cell Sorter at $\lambda_{\text {exc }}=488 \mathrm{~nm}$ and allowed to grow to confluency before splitting them in triplicate plates to screen for mutant clones.

Clones were lysed in 96 -well plates using $50 \mu \mathrm{l}$ radioimmunoprecipitation assay buffer ( $150 \mathrm{mM} \mathrm{NaCl}, 50 \mathrm{mM}$ Tris HCL, pH 8.0, $1.7 \%$ NP40, $0.17 \%$ SDS, $0.5 \%$ Na-deoxycholate, 5 mM EDTA) supplemented with Protease inhibitor cocktail (Roche). Samples were clarified by centrifugation and analyzed using immunoblot technique.

\section{Sedimentation Velocity Analysis}

Sedimentation velocity experiments were carried out using a Beckman Optima XL-I analytical ultracentrifuge (Beckman Coulter) equipped with an eight-position AN-60Ti rotor. The protein samples $(25 \mu \mathrm{M})$ were loaded in the cells, using, in all cases, the reference buffer (PBS). Samples in double-sector cells were centrifuged at $40,000 \mathrm{rpm}$. For all experiments, temperature was kept at $20^{\circ} \mathrm{C}$. Sedimentation profiles were recorded using interference scanning optics. For analysis of the data, the program Sedfit was used to calculate sedimentation coefficient distribution profiles using the Lamm equation (Schuck, 2000).

\section{GST Pull-Down Assay}

Recombinant GST-CtBP2 was produced in BL21 (DE3)-RIL competent cells following treatment of cells with $0.5 \mathrm{mM}$ isopropyl$\beta$-D-thiogalactopyranoside for 5 hours, lysed, and incubated with glutathione-agarose beads (Thermo Scientific) for 2 hours in immunoprecipitation (GST-IP) buffer consisting of $20 \mathrm{mM}$ HEPES, $\mathrm{pH}$ 7.3, $200 \mathrm{mM} \mathrm{NaCl}, 0.5 \mathrm{mM}$ EDTA, 1\% IGEPAL CA-630 (octylphenoxypolyethoxyethanol), $0.5 \mathrm{mM}$ DTT, and protease inhibitors (Roche) (added just prior to the experiment). After binding of protein to the beads and thorough washing $(4 \times)$, the modified beads were incubated with relevant recombinant proteins (glycerol-free) overnight at $4^{\circ} \mathrm{C}$ in GST-IP buffer. After binding of protein to the beads and thorough washing $(4 \times)$ following that, resin-bound proteins were eluted by heating, resolved by SDS-PAGE, and detected by IB with anti-GST and anti-His antibodies.

\section{In Vivo Cross-Linking}

Disuccinimidyl glutarate (DSG) cross-linker was purchased from Thermo Scientific and appropriately stored with desiccants at $-20^{\circ} \mathrm{C}$. A stock solution of 10 or $50 \mathrm{mM}$ in DMSO was made fresh for each experiment. In vivo cross-linking was carried out in cells that were collected by scraping, washed with PBS, and later resuspended in PBS ( $\mathrm{pH} \sim 8.2$ ) with $1 \times$ Complete Protease Inhibitor Mixture, EDTA-free (Roche Applied Science). Samples were incubated with cross-linker $(250 \mu \mathrm{M})$ for 30 minutes at $37^{\circ} \mathrm{C}$ with rotation. The reaction was quenched with the addition of $1 \mathrm{M}$ Tris, $\mathrm{pH} 7.6$, to $20 \mathrm{mM}$ final concentration and incubated for 15 minutes at room temperature. After quenching, in vivo cross-linked samples were lysed using radioimmunoprecipitation assay buffer, followed by ultracentrifugation for 30 minutes at $15,000 \mathrm{rpm}$ at $4^{\circ} \mathrm{C}$. Samples were then loaded and analyzed on SDS-PAGE gel for efficiency of interprotein crosslinking.

\section{Immunoprecipitations}

For IP experiments, HCT116; $\mathrm{CtBP} 2^{-/-}$cells were transfected with expression vector that encodes either vector, wt, or relevant mutants (6 $\mu \mathrm{g}$ ) using lipofectamine 2000, as recommended by the manufacturer (Invitrogen). After 48 hours, cells were lysed in IP buffer (20 mM Tris, $\mathrm{pH} 7.5,150 \mathrm{mM} \mathrm{NaCl}, 1 \% \mathrm{NP} 40$, protease inhibitor-added fresh). Whole-cell lysates (1.0-2.0 mg) were precleared by incubation with protein G Agarose Fast Flow beads (EMD Millipore) for 1 hour on a rotator at $4^{\circ} \mathrm{C}$. Two micrograms of relevant antibodies were added to 
the precleared lysates and incubated on a rotator at $4^{\circ} \mathrm{C}$ for 1 hour. Then washed beads were added to these mixtures and incubated overnight. Following incubation, beads were washed three times in IP buffer, resuspended in $40 \mu \mathrm{l}$ of $1 \mathrm{X}$ loading buffer (NuPage-Invitrogen), heated to $95^{\circ} \mathrm{C}$ for 10 minutes to isolate protein and beads, and then centrifuged for clear supernatant, which was then analyzed by SDSPAGE using standard Western blotting technique. For endogenous drug assays, the cells were treated with vehicle, 500 or $1000 \mu \mathrm{M}$ inhibitor for 24 hours before cellular lysis, post-transfection of either $\mathrm{EV}$ or wt CtBP2, and the IP protocol was followed.

\section{Reverse-Transcription Polymerase Chain Reaction and Real-Time Reverse-Transcription Polymerase Chain Reaction}

Forty-eight hours post-transfection of $6 \mu \mathrm{g} \mathrm{EV}$, wt, G189A, R272A, $\mathrm{W} 324 \mathrm{~F}$, and W324A plasmids, total cellular RNAs were isolated from samples using QIAGEN RNeasy kit and instructions therein. Later, cDNA synthesis was carried out using SensiFAST cDNA Synthesis Kit from BIOLINE. Quantitation of all gene transcripts was done by quantitative polymerase chain reaction (qPCR) using SYBR Green (Applied Biosystems, Foster City, CA) and an ABI 7300 (Applied Biosystems) machine.

18srRNA expression was used as an internal control. The primer pairs used were as follows: 18srRNA, 5'-CGCCGCTAGAGGTGAAATTC-3' (forward) and 5'-TGGCAAATGCTTTCGCTCTG-3' (reverse); TIAM1, 5' CGCTGGAGTCGTACCTCATC-3' (forward) and 5'-GGTCAAACACAGCCCCAAAC-3' (reverse). Relative amounts of the mRNA transcripts were calculated using the $\Delta \Delta \mathrm{CT}$ method and reported as fold change with respect to EV transfection (Schmittgen and Livak, 2008).

\section{In Vitro Wound-Healing Assay (Mutant and Inhibitor)}

Mutants. MDA-MB-231;CtBP2-/- cells were seeded into six-well dishes at a density of $5 \times 10^{5}$ cells/well. The dishes were cultured as confluent monolayers and were then further transfected with $3 \mu \mathrm{g} \mathrm{EV}$, wt CtBP2, G189A, R272A, W324F, and W324A expression plasmids.

Inhibitor. MDA-MB-231 cells (CtBP2 wt) were seeded into sixwell dishes at a density of $5 \times 10^{5}$ cells/well. The dishes were cultured as confluent monolayers and were then further treated with 4-Cl HIPP at $156,312,625,1250$, and $2500 \mu \mathrm{M}$ concentration, whereas a vehicletreated dish served as control.

Following 24-hours of either transfection or drug incubation, a scratch was made once per well with a $200-\mu l$ pipette tip to create an artificial wound. Wounded cell cultures were then incubated in the presence of DMEM after thorough, but gentle washes. The migration of cells was monitored over a duration of 24 hours for transfection and 24,40 , and 48 hours for inhibitor treatment, as a function of how far from the scratch line the cells had progressed. The scratch closures were quantified using ImageJ (NIH). The area at a time point is normalized relative to 0 -hour time and reported as absolute value.

\section{Data Analysis and Statistical Methods}

All experiments were carried out three times (represented as $N=3$ in their legends). Student $t$ test was used for statistical analysis of Fig. 3B and Supplemental Fig. 6. Statistical evaluation of the differences between the groups from the qPCR and mutant CtBP2 migration data (Fig. 5, A and B) was conducted initially using a one-way analysis of variance, followed by multiple comparisons with false discovery rate (FDR) adjustments for handling multiplicity issues (Benjamini and Hochberg, 1995). The FDR adjustment is usually a less stringent control of type-1 error, compared with family-wise error controls, via the Bonferroni adjustment, leading to higher power to detect truly significant results. For the migration data of inhibitortreated cells (Fig. 5C), where measurements were taken at four time points, we initially conducted a one-way repeated-measures analysis of variance, followed by FDR-adjusted multiple comparisons to test group differences. We used SAS statistical software, Version 9.4 of the
SAS System for Windows, for all analyses. The error bars refer to S.D. for all calculations. Note: For Fig. 5A, qPCR analysis yielded statistically significant differences when comparisons were made between TIAM1 transcription driven by wt CtBP2 versus CtBP2 mutants, within two $(N=2)$ initial repeats of the experiment. Based on these findings after two repeats of the experiment, we determined that one additional repeat of the experiment for an $N=3$ would provide adequate statistical power to test the significance of the disruption of TIAM1 transactivation by CtBP2 mutations.

\section{Results}

Active-Site Tryptophan 324 Contributes to CtBP2 Self-Association That Can Be Abrogated by 4-Cl HIPP In Vitro. To understand the role of W324 in CtBP function, we first investigated the effect of W324 mutation on CtBP2's self-association capability, focusing on CtBP2, as the CtBP1 and CtBP2 dehydrogenase domains are structurally nearly identical (Hilbert et al., 2014). Oligomerization of CtBP contributes directly toward CtBP's transcription coregulatory activity (Dcona et al., 2017). The efficiency of oligomerization is intensified in the presence of $\operatorname{NAD}(\mathrm{H})$, which further influences CtBP's ability to associate with other coregulators (Bhambhani et al., 2011; Bellesis et al., 2018). Previous studies have shown that mutations in the CtBP1 nucleotidebinding domain (NBD) diminish CtBP1's ability to oligomerize and also influence its transcriptional activities (Mani-Telang et al., 2007). Having previously engineered a His-tagged truncated version of wt CtBP2 protein (Fig. 1B) (Hilbert et al., 2014) active for dehydrogenase and oligomerization activities, we compared the biophysical and functional effects of a series of W324 mutations to control mutations known to directly affect CtBP nucleotide binding (G189A) or catalysis (R272A and H321A) (Dcona et al., 2017). W324 was then mutated to G, A, and F (Fig. 1C): W324F partially tests the requirement for the indolyl ring at position 324 , as opposed to a phenyl ring, which is predicted to also engage in a paralleldisplaced $\pi-\pi^{*}$ interaction with substrate bound within the catalytic domain (McGaughey et al., 1998); W324A and G are hydrophobic mutations that should plausibly abrogate CtBP2 dehydrogenase activity by weakening the affinity for substrate, as $\pi-\pi^{*}$ interaction of the MTOB thioether with the 324 residue would not be possible, with W324G further testing the effect of conformational freedom not available in W324A (de Groot et al., 1997).

Prior work has demonstrated that truncated CtBP2(31-364) (st. CtBP2) dimerizes in accordance with crystallographic data, whereas, in solution, full-length CtBP1 tetramerizes as a dimer of dimers (Kumar et al., 2002; Madison et al., 2013). For the purpose of our studies, we used st. CtBP2, due to the highly unstructured nature of the $\mathrm{C}$ terminus of $\mathrm{CtBP} 1 / 2$ arising from a proline/glycine-rich sequence (Nardini et al., 2006). Hence, for the purpose of simplicity and to maintain consistency with previous reports (Kumar et al., 2002; Hilbert et al., 2014, 2015), in vitro studies used the residue 31-364 truncated versions of wt and mutant (mt) CtBP2 recombinant proteins.

After affinity-based purification, the oligomeric behavior of wt and mt His-tagged st. CtBP2 proteins was analyzed by analytical ultracentrifugation (AUC) using sedimentation velocity. The sedimentation coefficient distribution of the wt protein at $27.2 \mu \mathrm{M}$ indicated the presence of a predominant dimeric population ( $\mathrm{s}=6-6.7$ ), which was further intensified 
in the presence of $10 \mu \mathrm{M} N A D(H)$ (Fig. 2, A-C). The range of values representing the CtBP monomer/dimer species is a result of the presence of the His-tag and the multiple conformations that it can acquire. The values are consistent with the theoretical sedimentation velocity coefficients calculated using the CtBP2 crystal structure and two different conformations of His-tail using the program SOMO as part of the ultrascan III (Schuck, 2000; Demeler, 2010). Mutations in any relevant functional domain of CtBP2 (G189A, R272A, H321A; see Fig. 1C) impaired self-association, resulting in a mixed population of monomer and dimer (Fig. 2, D and E; Supplemental Fig. 1) (Kumar et al., 2002; Madison et al., 2013). Interestingly, W324F and W324G mutants exhibited monomeric or mixed monomer/dimer populations without $\mathrm{NAD}(\mathrm{H})$, but yielded patterns similar to that of wt in the presence of $\mathrm{NAD}(\mathrm{H})$. In contrast, W324A was primarily
( $\sim 60 \%)$ monomeric, despite addition of $\mathrm{NAD}(\mathrm{H})$ (Fig. 2, D and E; Supplemental Fig. 1). Taken together, these results demonstrate that the indolyl moiety of the W324 residue is pivotal for CtBP2 oligomerization, and suggest that this residue functions to stabilize the oligomeric interface (Madison et al., 2013).

Given the strong interaction of the substrate-competitive CtBP inhibitor HIPP with W324 (Hilbert, et al., 2015), we examined the effect of the more potent second-generation CtBP inhibitor 4-Cl-HIPP (Korwar et al., 2016) on CtBP2 selfassociation, to determine whether disruption of the oligomer could be part of this inhibitor's mechanism of action. For this purpose, CtBP2 was incubated with increasing concentration of 4-Cl-HIPP (10-1000 $\mu \mathrm{M})$ with $10 \mu \mathrm{M} \mathrm{NAD}(\mathrm{H})$, and the oligomerization state was probed using sedimentation velocity. Surprisingly, only $10 \mu \mathrm{M}$ 4-Cl-HIPP (only 58.4-fold higher
A
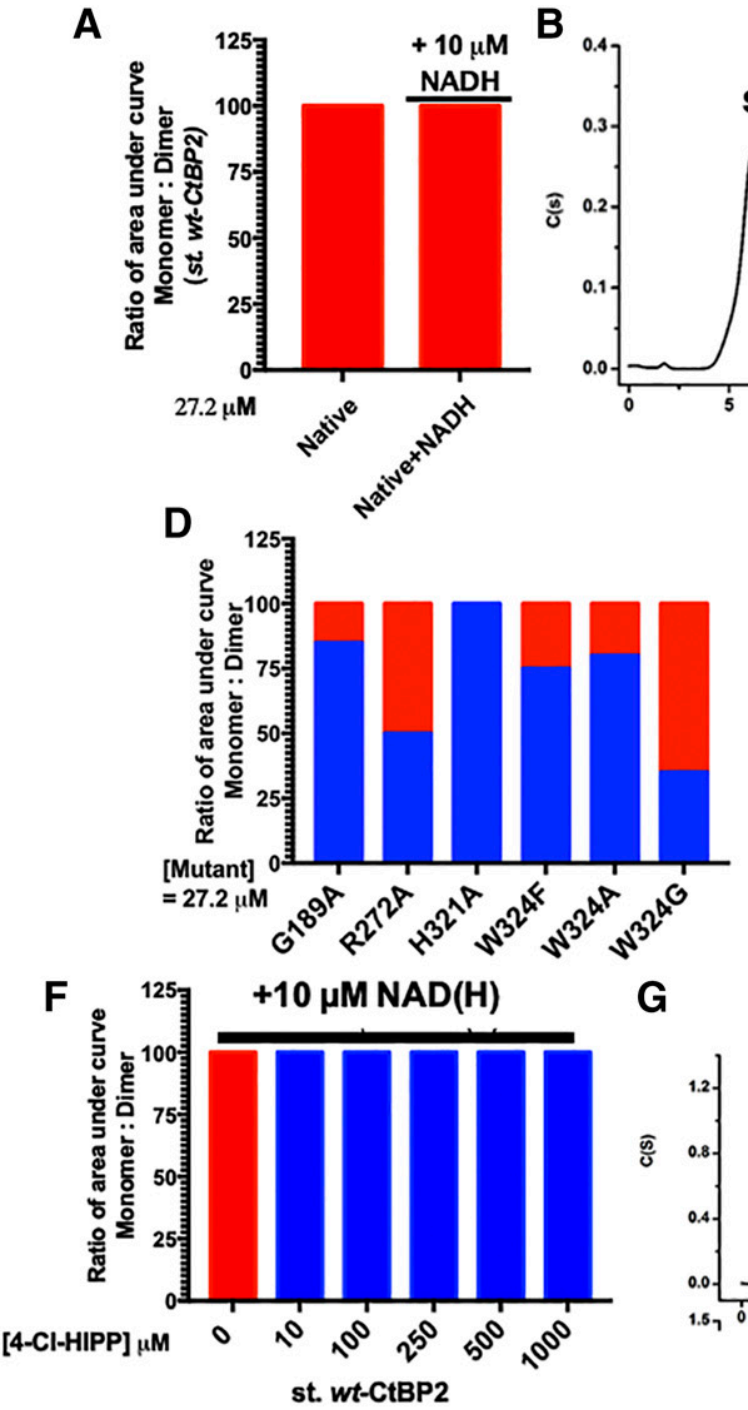

G
B

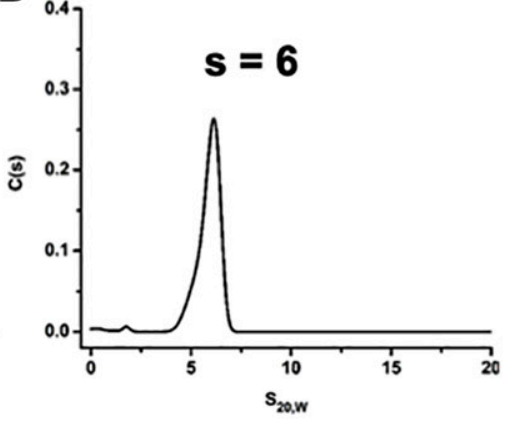

C

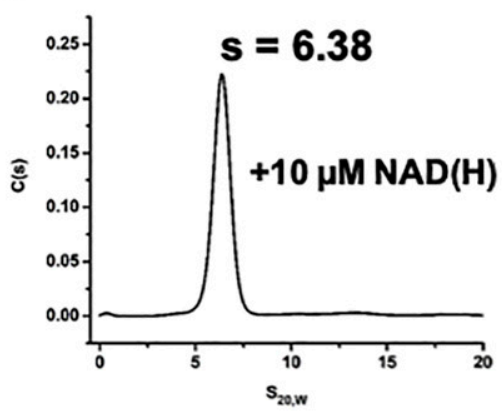

E

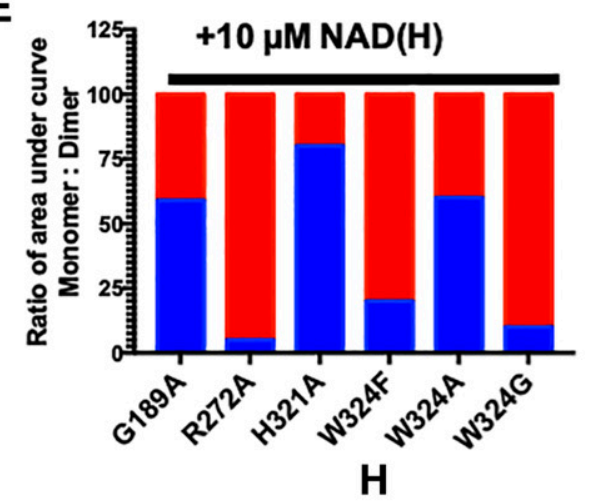

$s=\mathbf{5 . 8 2}$
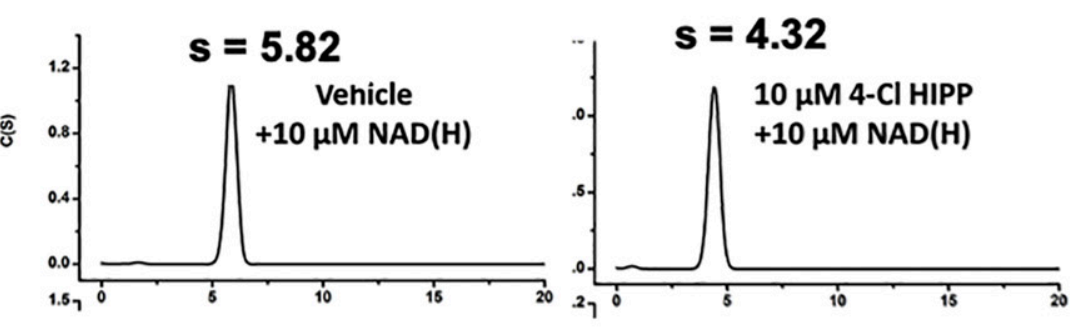

Monomer

Dimer

Fig. 2. (A-E) Normalized ratio of area under the curve for monomer and dimer calculated using SedFit and sedimentation coefficient distribution profile of native wt CtBP2 (A and B) or mt CtBP2 (D); and wt CtBP2 (A and C) or mt CtBP2 (E) in the presence of $10 \mu \mathrm{M} \mathrm{NAD}(\mathrm{H})$. (F) Graphical representation of area under the curve for AUC assay of wt CtBP2 incubated with varying 4-Cl-HIPP concentrations and $10 \mu \mathrm{M} \mathrm{NAD}(\mathrm{H})$. (G and H) Sedimentation coefficient distribution of wt CtBP2 incubated with $10 \mu \mathrm{M} N A D(H)(G)$ or $10 \mu \mathrm{M}$ 4-Cl-HIPP and $10 \mu \mathrm{M}$ NAD $(\mathrm{H})(\mathrm{H})$. The protein concentration for each assay was $27.2 \mu \mathrm{M}$. Red, monomer; blue, dimer. 
than the $K_{\mathrm{d}}$ for HIPP/CtBP interaction) was sufficient to robustly disrupt CtBP2 oligomerization, indicating that HIPP-class CtBP dehydrogenase inhibitors disrupt not only catalysis, but also CtBP2 homodimerization (Fig. 2, F-H; Supplemental Fig. 2) (Korwar et al., 2016).

W324 Mutation Disrupts Self-Association with WildType CtBP2. To further illustrate that W324 is important for CtBP2's self-association, we investigated the ability of $\mathrm{mt} \mathrm{CtBP} 2$ to heterodimerize with wt CtBP2 in the presence of $\mathrm{NAD}(\mathrm{H})$, using a glutathione-S-transferase (GST)-based pull-down assay. GST-CtBP2 (31-364) protein was immobilized onto glutathione-agarose beads, and incubated with wt or mt CtBP2 proteins tagged with His6 epitope at different concentrations (Fig. 3, A and B). As predicted by the AUC homodimerization analyses, dimerization between two differently tagged wt CtBP2 proteins proceeded as expected, and the interaction increased with an increase in the concentration of His6-wt-CtBP2 protein (Fig. 3A). In contrast, Histagged CtBP2 W324A and G189A mutant proteins were both similarly defective for heterodimerization with wt-GSTCtBP2 as evidenced from the blots and densitometry calculations (Fig. 3, A and B; Supplemental Fig. 3).

Determination of $\mathrm{IC}_{50}$ for 4-Cl-HIPP Disruption of Oligomerization. After understanding that W324A impairs heterodimerizing capability in the pull-down assay, and knowing that 4-Cl-HIPP can disrupt CtBP oligomerization at or near its $K_{\mathrm{d}}$, we used the pull-down assay to more quantitatively measure the $\mathrm{IC}_{50}$ for disruption of oligomerization of 4-Cl-HIPP versus an inactive (for catalytic inhibition) control HIPP derivative, 2-methoxy-HIPP (Fig. 3C; Supplemental Fig. 4) (Korwar et al., 2016). Using log-scale titration of 4-Cl-HIPP from 0.01 to $500 \mu \mathrm{M}$, we found that
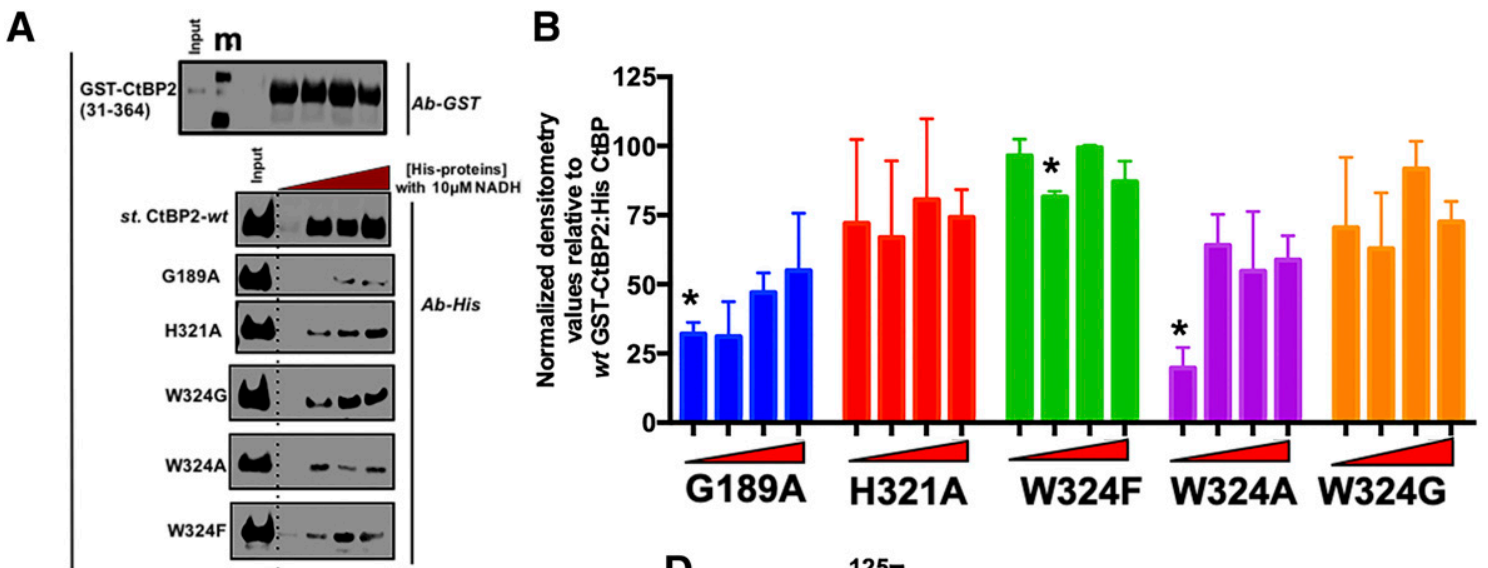

C
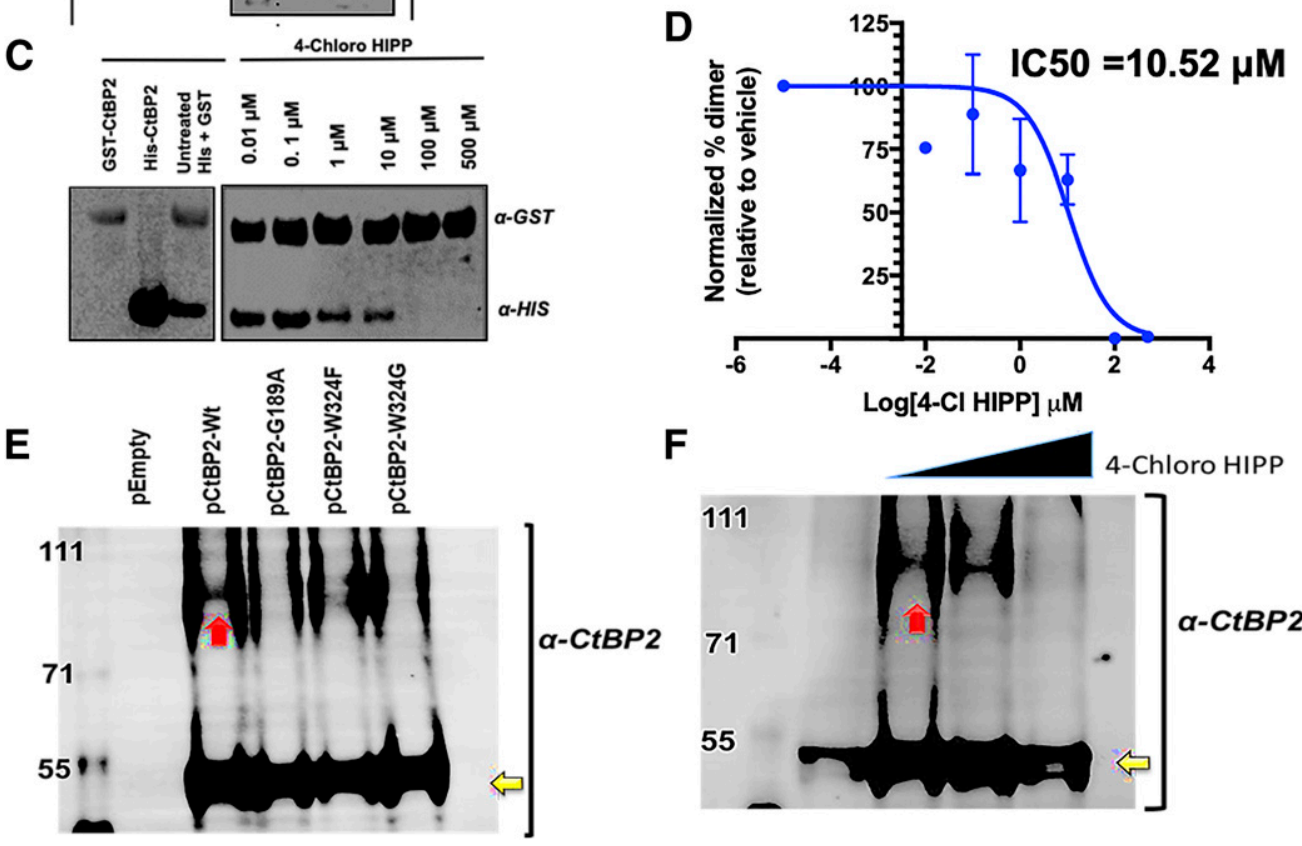

Fig. 3. (A-D) GST pull-down assay using GST-CtBP2(31-364) incubated with (A and B) wt or mt His-st. CtBP2 and $10 \mu \mathrm{M}$ NAD(H) or (C and D) wt Hisst. CtBP2 incubated with increasing doses of 4-Cl-HIPP and $10 \mu \mathrm{M} N \mathrm{NAD}(\mathrm{H})$ with pulldowns and input purified GST- and His- CtBP2 proteins immunoblotted with indicated antibodies. The letter "m" in (A) on top of the representative immunoblot for GST-wt-CtBP2 denotes the lane containing protein molecular weight standards and ${ }^{*} P$ value $<0.05$. Triangles indicate increasing dosage of wt or mt proteins $(N=3)$. (E) HCT116;CtBP2 ${ }^{-1-}$ cells were transfected with empty vector or either wt or mt CtBP2 expression plasmids, and cells were treated with DSG for 30 minutes, followed by lysis and CtBP2 immunoblot. (F) HCT116;p53-/- cells expressing endogenous wt CtBP2 were treated with vehicle or 4-Cl HIPP at 500 and $1000 \mu \mathrm{M}$ for 24 hours (lanes 3-5), and cells were then exposed to DSG for 30 minutes, followed by lysis and CtBP2 immunoblot $(N=3)$. 1st lane represents protein molecular weight markers and 2nd lane represents cells untreated with either 4-Cl HIPP or DSG. Yellow arrow represents monomer (mol. wt. $49 K_{\mathrm{D}}$ ), and red arrow indicates dimer (mol. wt. $\sim 98 K_{\mathrm{D}}$ ). ${ }^{*} P<0.05$ (Student $t$ test). 
CtBP2 dimerization diminished between 1 and $10 \mu \mathrm{M} 4-\mathrm{Cl}$ HIPP and disappeared after further titration of the drug above $10 \mu \mathrm{M}$ (Fig. 3C). The calculated $\mathrm{IC}_{50}$ value of inhibition of dimerization by 4-Cl-HIPP was $10.52 \mu \mathrm{M}$ (Fig. 3D), consistent with AUC analyses above (Supplemental Fig. 2), whereas 2-methoxy-HIPP displayed an $\mathrm{IC}_{50}$ for disruption of heterodimerization $>100 \mu \mathrm{M}$ (Supplemental Fig. 4) (Korwar et al., 2016). Thus, in addition to inhibiting CtBP's dehydrogenase activity, 4-Cl HIPP acts as a high-affinity inhibitor of CtBP oligomerization, which is required ultimately for its transcriptional function (Dcona et al., 2017).

Cross-Linking of CtBP2 In Vivo Reveals That W324 Is Central to the Formation of Physiologic Oligomeric Species That Can Be Disrupted Using 4-Cl HIPP. To analyze the physiologic oligomeric state of full-length CtBP2 in situ in living cells, we next performed chemical protein cross-linking of whole cells in culture (Fig. 3E; Supplemental Fig. 5). For this purpose, we transfected expression plasmids encoding wt, G189A, W324F, and W324G-mutated CtBP2 cDNA into HCT116 cells homozygously deleted for CtBP2 using CRISPR techniques (Chawla et al., 2018). Intact transfected cells were then incubated with DSG at $37^{\circ} \mathrm{C}$ for 30 minutes, and total cellular protein was immunoblotted for CtBP2. CtBP2 was absent, as expected, from vectortransfected cells, and wt CtBP2 exhibited distinct forms consistent with monomer and dimer based on predicted molecular weight (Fig. 3E). Notably, W324F and G proteins exhibited a lower efficiency of dimer and higher oligomer formation as did G189A, consistent with our in vitro AUC and pull-down data (Figs. 2 and 3). All of the above results indicate that W324 is an essential residue that contributes significantly toward CtBP2's oligomeric capability, including in the setting of full-length CtBP2 in the physiologic cellular environment.

After understanding that the oligomer formation of CtBP2 is strongly dependent on W324, we performed a chemical cross-linking experiment using DSG in HCT116;p53-/- cells with wt CtBP2 expression were treated with either 500 or $1000 \mu \mathrm{M} 4$-Cl HIPP. Here again (Fig. 3F), we found that the ability of CtBP2 to dimerize was abrogated in the presence of the 4-Cl-HIPP, similar to the effect of the drug in vitro in the AUC and pull-down experiments, albeit at higher concentration, due presumably to either poor cellular penetration or rapid intracellular metabolism.

Interaction of CtBP2 with Other Transcriptional Coregulators Is Facilitated by W324. CtBP associates with CoREST among other chromatin modifier proteins to transcriptionally coregulate (repress and activate) numerous cancer-related tumor suppressors and oncogenes, including CDH1 and EGFR (Quinlan et al., 2006; Li et al., 2017). Having established that W324 influences CtBP2's oligomerization capabilities, we further inquired whether physiologic functions, such as CtBP coassociation with CoREST (Choi et al., 2013), depend on intact W324. For this purpose, plasmids encoding wt-CtBP2, CtBP2-G189A, and CtBP2-W324G were transfected into HCT116; $\mathrm{CtBP} 2^{-/-}$cells along with $\mathrm{EV}$ control, and, 24 hours post-transfection, cell lysates were immunoprecipitated with anti-CtBP2 antibody, followed by IB with antibodies against CtBP2, CoREST, and vinculin (loading control). Whereas wt CtBP2 exhibited robust coimmunoprecipitation with CoREST, neither the W324G nor G189A mutants were able to coimmunoprecipitate CoREST (Fig. 4A). Despite the

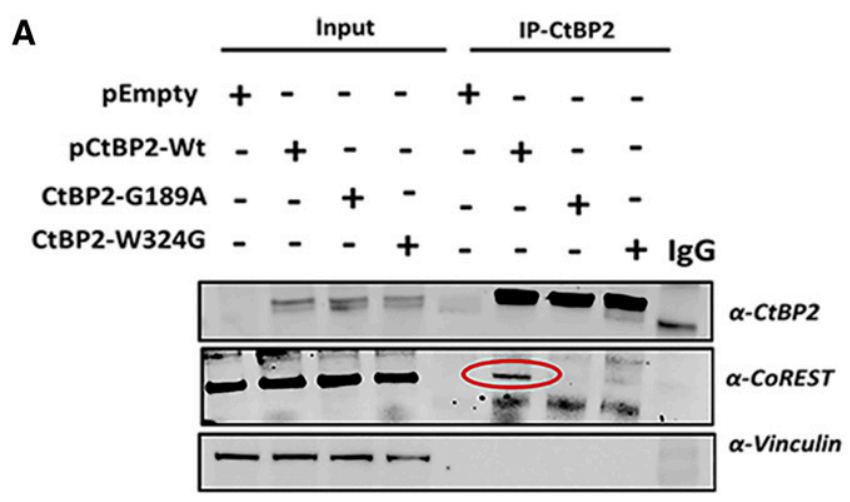

B

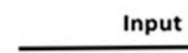

Input
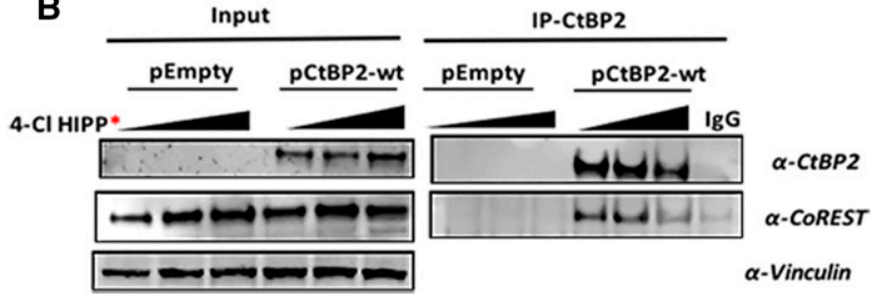

Fig. 4. Coimmunoprecipitation between CoREST and CtBP2 derived from lysates of HCT116;CtBP2-/- cells transfected with (A) wt or mt CtBP2 or (B) empty vector or wt CtBP2 plasmids. In (B), transfected cells were treated with vehicle, 500 or $1000 \mu \mathrm{M} 4$-Cl-HIPP. Input lysates and IP's were immunoblotted with indicated antibodies $(N=3)$.

close sequence similarity of CtBP1 and CtBP2, these data do, however, conflict with prior work showing that a cluster of mutants in the CtBP1 NBD, namely G183A and G186 (G189A and G192A in CtBP2), did not have any influence on the interaction between CtBP1 and CoREST (Kuppuswamy et al., 2008). Nevertheless, mutations that have in common disruption of CtBP2 oligomerization all disrupt CoREST/CtBP2 interaction, and these data may point to heretofore unknown distinct functions for CtBP1 and CtBP2 in transcriptional regulation, similar to distinct functions already described in Golgi regulation, mitosis, and ribbon synapse formation (Dcona et al., 2017).

Given the strong influence of W324 on CtBP2/CoREST interaction, we further investigated whether 4-Cl HIPP was able to disrupt CtBP2/CoREST complex formation. Twentyfour hours post-transfection of wt-CtBP2 or EV, HCT116; $\mathrm{CtBP}^{-/-}$cells were incubated with 500 and $1000 \mu \mathrm{M} 4-\mathrm{Cl}$ HIPP for an additional 24 hours, and cell lysates were immunoprecipitated with anti-CtBP2 antibody, followed by IB with antibodies against CtBP2, CoREST, and vinculin (loading control). The 4-Cl HIPP at $1000 \mu \mathrm{M}$, but not $500 \mu \mathrm{M}$, effectively disrupted CtBP2/CoREST complexation in a manner similar to the disruption provided by mutation of W324 to glycine (Fig. 4B). Thus, genetic or pharmacologic targeting of CtBP2 W324 inactivated a key biochemical correlate of its transcriptional activity, namely interaction with a member of the CtBP transcriptional supercomplex (Shi, et al., 2003).

W324 Mutation or Inhibitor Abrogates CtBP2 Transcriptional Regulation and Induction of Cellular Motility. A key pro-oncogenic function of CtBP is to induce cell migratory behavior that is related to CtBP's role in induction of epithelial-mesenchymal transition and metastasis (Dcona, et al., 2017). Previously, we have shown that CtBP2 activates the TIAM1 gene that mediates CtBP-dependent cell 
migration (Minard et al., 2004; Paliwal et al., 2012). Therefore, we inquired whether intact W324 is required for CtBP2 to transactivate TIAM1 and also affect cell migration. Plasmids encoding vector control, wt CtBP2, or CtBP2 mutants G189A, R272A, and W324F/A were transfected into HCT116; $\mathrm{CtBP}^{-/-}$cells, followed by extraction of total mRNA and qPCR determination of TIAM1 mRNA abundance. Expression of wt and $\mathrm{mt}$ CtBP2 proteins was equivalent, as determined by CtBP2 immunoblot (Supplemental Fig. 6). Whereas wt CtBP2 activated TIAM1 4.5-fold, all CtBP2 mutants were defective for TIAM1 induction (Fig. 5A). This effect could be explained by disruption of oligomerization, where monomeric CtBP2 mutant proteins are unable to form the active CtBP transcriptional supercomplex that bridges chromatin-modifying enzymes with DNA-interacting transcription factors (Dcona et al., 2017).

Next, we investigated whether cellular motility is affected by mutation of W324. MDA-MB-231; $\mathrm{CtBP}^{-1-}$ (CRISPR deletion) cells were transfected with vector, wt-CtBP2, or CtBP2 G189A, R272A, or W324F/A plasmids, and after 24 hours, a scratch was made and cells were allowed to migrate to fill the scratch. Expression of wt and mt CtBP2 proteins was equivalent, as determined by CtBP2 immunoblot (Supplemental Fig. 7). Whereas wt CtBP2 transfection significantly accelerated wound closure within 24 hours, all mutants were significantly defective for promoting wound closure, indicating that W324, CD, and NBD are all required for both TIAM1 transactivation and the key physiologic cellular CtBP function of promoting migration (Fig. 5B).

To chemically phenocopy genetic inactivation of $\mathrm{W} 324$, MDA-MB-231;CtBP2-/- cells were treated with 0-2500 $\mu \mathrm{M}$ 4-Cl HIPP, followed by a scratch assay time course over 48 hours. With increasing dosage of the inhibitor, the ability of cells to migrate diminished, and then nearly ceased, at the highest dosage, despite minimal loss of cell number during this time frame (Fig. 5C). Thus, pharmacologic targeting of W324 and catalytic function impairs CtBP2 oligomerization, transactivation of a key migration gene, and ultimately CtBP2's ability to effectively drive cell migration.

\section{Discussion}

In an attempt to understand the reasons behind the antineoplastic efficacy of CtBP dehydrogenase inhibitors, we have analyzed the contribution of the key inhibitor-interacting residue, CtBP2 W324 (Madison et al., 2013; Hilbert et al., 2015), to oligomerization and key biochemical/functional

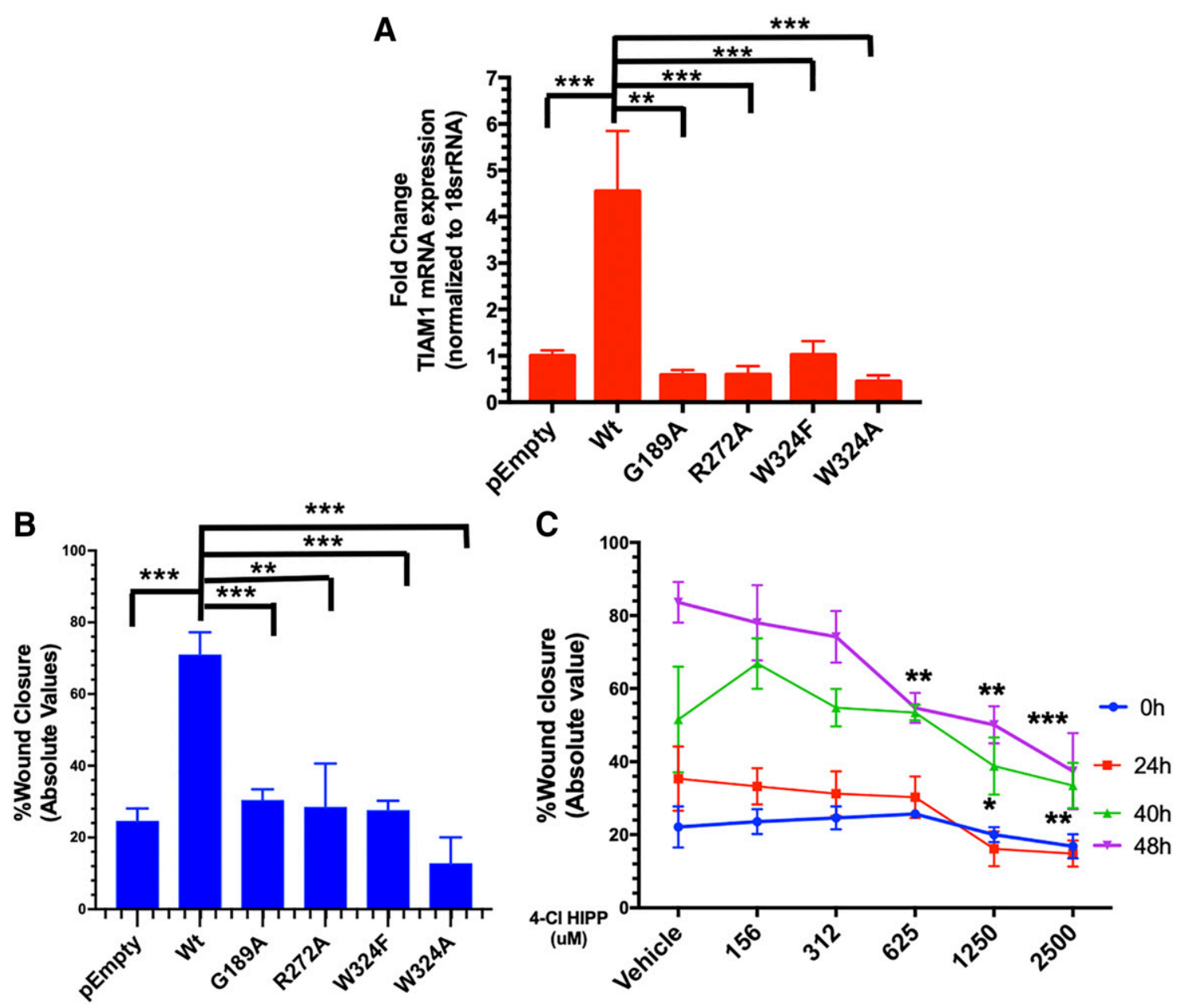

Fig. 5. (A) Fold change of TIAM1 mRNA expression after transfection of either wt or mt CtBP2, relative to EV transfection $(N=3)$. (B and C) Graph representing quantified area of scratch after 24 hours measured relative to area at 0-hour time point in cells transfected (B) with either wt or mt CtBP2 $(N=3)$, or $($ C) with wt CtBP2 and treated with 4-Cl HIPP at concentrations between 0 and $2500 \mu \mathrm{M}$ for 24 hours $(N=3)$. False discovery rate procedure was applied, and pairwise comparisons were conducted to determine the significance of these experiments. ${ }^{*} P<0.05 ; * * P<0.01 ; * * * P<0.001$. 
correlates of transcriptional complex formation, including activation of a key migration gene and cell migration. Prior work reported that the paralogous CtBP1 W318 residue acts as a switch that links $\mathrm{NAD}(\mathrm{H})$ binding to dimerization through a strand exchange mechanism, which then facilitates tetramer (dimer of dimer) formation (Madison et al., 2013). Of note, in this work, F.L. CtBP1 (W318F) exhibited diminished ability to form tetramers, but dimerized partially (Madison et al., 2013). Our data corroborate and extend these results, wherein we witnessed that mutation or chemical targeting of CtBP2 W324 impaired dimerization as observed from altered sedimentation coefficients and pull-down assays.

It is worth noting that, despite extensive purification, the wt CtBP2 protein existed as a dimer in the absence of added $\mathrm{NAD}(\mathrm{H})$, as evidenced from AUC (Fig. 2A), which could be due to the presence of $\operatorname{NAD}(\mathrm{H})$ nucleotide from $E$. coli, whereas some mt CtBP2 proteins existed as mixed populations of monomer and dimer and underwent conformational rearrangement in the presence of excess nucleotide (Fig. $2 \mathrm{E}$ ). Of note, the H321A, G189A, and W324A mutants remained largely unaltered in oligomeric state despite the addition of $\mathrm{NAD}(\mathrm{H})$, which could be due to an impaired strand-exchange oligomerization mechanism. Contrary to our expectations, wherein we assumed that all nonaromatic mutants of W324 would have impaired oligomerizing capabilities, W324G exhibited a substantial dimer population, even in the absence of the $\operatorname{NAD}(\mathrm{H})$. Therefore, it is reasonable to suppose that there could be an equilibrium between monomeric and dimeric populations of CtBP2 W324G protein, as is the case presumably for $\mathrm{W} 324 \mathrm{~F}$, which, although impaired for dimerization in the absence of NADH, readily dimerized with $\mathrm{NADH}$ addition. Exactly why W324G dimerizes, whereas other nonaromatic mutants are impaired, is unclear, and will require further in silico modeling and/or X-ray crystallographic analysis of the W324G dimer.

Consistent with our previous hypothesis that the HIPP-CtBP complex may be trapped in an inactive abortive ternary complex (Hilbert et al., 2015), we found that addition of increasing concentrations of 4-Cl-HIPP inhibited oligomerization of monomeric $\mathrm{CtBP} 2$, despite the presence of $\mathrm{NAD}(\mathrm{H})$. This is explained by the 4-Cl HIPP-CtBP-NAD $(\mathrm{H})$ complex assuming a closed conformation at 4-Cl-HIPP concentrations $\geq \sim 10$ $\mu \mathrm{M}$, the $\mathrm{IC}_{50}$ for $\mathrm{CtBP} 2$ oligomer disruption.

Mutation of CtBP2 W324, or substrate competitive inhibition with 4-Cl-HIPP, debilitated CtBP2 coassociation with CoREST (Cowger et al., 2007; Kuppuswamy et al., 2008; Choi et al., 2013; Li et al., 2017). Interestingly, mutation of W324 and the CtBP2 nucleotide binding (NBD) and catalytic (CD) domains disrupted TIAM1 transcription and migration. Specifically, transcriptional analysis using qPCR showed that the robust transcriptional induction of TIAM1 by CtBP2 was abolished by mutations W324A and $\mathrm{W} 324 \mathrm{~F}$ as well as the NBD and CD mutations G189A and R272A (Fig. 5A). Consistent with a key role of TIAM1 expression in the cellular migration phenotype, the same mutations also abolished the robust induction of cell migration observed with wt CtBP2 (Fig. 5B). Although in both the TIAM1 and migration assays certain mutants seemed to cause a possible dominantnegative effect with measured values going below control baseline values (Fig. $5, \mathrm{~A}$ and $\mathrm{B}$ ), the experiments were not sufficiently statistically powered to test this possibility, which will be explored in the future. Intriguingly, possible dominant-negative effects were particularly seen in the mutants that displayed diminished self-association characteristics not restorable with additional $\mathrm{NAD}(\mathrm{H})$, as evidenced from AUC and cross-linking experiments (Figs. 2 and 3), consistent with these specific mutations more potently disengaging the activities of CtBP dependent on oligomerization.

The dosage of 4-Cl-HIPP to achieve efficacy in cellular assays remains well beyond its in vitro effective concentration, and higher than desirable for a true therapeutic molecule. This need for high micromolar concentrations of inhibitor in vivo derives from the need to achieve stoichiometric excess over $\mathrm{NAD}(\mathrm{H})$ cellular concentrations, combined with likely inefficient cellular entry of 4-Cl-HIPP (Korwar et al., 2016). Using the mechanistic knowledge gained in this work, we are focused on creating more potent inhibitors that target W324 with improved cellular entry, to thus achieve therapeutic effect at doses within the range of practicality for clinical development.

\section{Acknowledgments}

We acknowledge John C. Hackett, Joe Landry, Matt Hartman (Virginia Commonwealth University Massey Cancer Center), and Warren D. Kruger (Fox Chase Cancer Center) for helpful suggestions.

\section{Authorship Contributions}

Participated in research design: Dcona, Grossman.

Conducted experiments: Dcona, Damle, Zarate-Perez, Morris, Nawaz, Dennis, Singh.

Contributed new reagents or analytic tools: Korwar, Ellis, Royer, Escalante.

Performed data analysis: Dcona, Deng, Bandyopadhyay, Escalante, Grossman.

Wrote or contributed to the writing of the manuscript: Dcona, Bandyopadhyay, Escalante, Grossman.

\section{References}

Barroilhet L, Yang J, Hasselblatt K, Paranal RM, Ng SK, Rauh-Hain JA, Welch WR, Bradner JE, Berkowitz RS, and Ng SW (2013) C-terminal binding protein-2 regulates response of epithelial ovarian cancer cells to histone deacetylase inhibitors. Oncogene 32:3896-3903.

Bellesis AG, Jecrois AM, Hayes JA, Schiffer CA, and Royer WE Jr (2018) Assembly of human C-terminal binding protein (CtBP) into tetramers. $J$ Biol Chem 293: 9101-9112.

Benjamini Y and Hochberg B (1995) Controlling the false discovery rate: a practical and powerful approach to multiple testing. J R Stat Soc Series B Stat Methodol 57: 289-300.

Bhambhani C, Chang JL, Akey DL, and Cadigan KM (2011) The oligomeric state of CtBP determines its role as a transcriptional co-activator and co-repressor of Wingless targets. EMBO $J$ 30:2031-2043.

Chawla AT, Cororaton AD, Idowu MO, Damle PK, Szomju B, Ellis KC, Patel BB, and Grossman SR (2018) An intestinal stem cell niche in Apc mutated neoplasia targetable by CtBP inhibition. Oncotarget 9:32408-32418.

Chinnadurai G, editor (2000) Unique transcriptional regulators in the nucleus with diverse cytosolic functions, in CtBP Family Proteins, Springer, New York.

Choi SH, Estarás C, Moresco JJ, Yates JR III, and Jones KA (2013) $\alpha$-Catenin interacts with APC to regulate $\beta$-catenin proteolysis and transcriptional repression of Wnt target genes. Genes Dev 27:2473-2488.

Cowger JJM, Zhao Q, Isovic M, and Torchia J (2007) Biochemical characterization of the zinc-finger protein 217 transcriptional repressor complex: identification of a ZNF217 consensus recognition sequence. Oncogene 26:3378-3386.

Dcona MM, Morris BL, Ellis KC, and Grossman SR (2017) CtBP - an emerging oncogene and novel small molecule drug target: advances in the understanding of its oncogenic action and identification of therapeutic inhibitors. Cancer Biol Ther 18:379-391.

de Groot BL, van Aalten DM, Scheek RM, Amadei A, Vriend G, and Berendsen HJ (1997) Prediction of protein conformational freedom from distance constraints. Proteins 29:240-251.

Demeler B (2010) Methods for the design and analysis of sedimentation velocity and sedimentation equilibrium experiments with proteins. Curr Protoc Protein Sci Chapter 7:Unit 7.13.

Hilbert BJ, Grossman SR, Schiffer CA, and Royer WE Jr (2014) Crystal structures of human CtBP in complex with substrate MTOB reveal active site features useful for inhibitor design. FEBS Lett 588:1743-1748.

Hilbert BJ, Morris BL, Ellis KC, Paulsen JL, Schiffer CA, Grossman SR, and Royer WE Jr (2015) Structure-guided design of a high affinity inhibitor to human CtBP. ACS Chem Biol 10:1118-1127. 
Korwar S, Morris BL, Parikh HI, Coover RA, Doughty TW, Love IM, Hilbert BJ, Royer WE Jr, Kellogg GE, Grossman SR, et al. (2016) Design, synthesis, and biological evaluation of substrate-competitive inhibitors of C-terminal binding protein (CtBP). Bioorg Med Chem 24:2707-2715.

Kumar V, Carlson JE, Ohgi KA, Edwards TA, Rose DW, Escalante CR, Rosenfeld MG, and Aggarwal AK (2002) Transcription corepressor CtBP is an $\mathrm{NAD}(+)$ regulated dehydrogenase. Mol Cell 10:857-869.

Kuppuswamy M, Vijayalingam S, Zhao LJ, Zhou Y, Subramanian T, Ryerse J, and Chinnadurai G (2008) Role of the PLDLS-binding cleft region of CtBP1 in recruitment of core and auxiliary components of the corepressor complex. Mol Cell Biol 28:269-281.

Li L, Liu X, He L, Yang J, Pei F, Li W, Liu S, Chen Z, Xie G, Xu B, et al. (2017) ZNF516 suppresses EGFR by targeting the CtBP/LSD1/CoREST complex to chromatin. Nat Commun 8:691-695.

Madison DL, Wirz JA, Siess D, and Lundblad JR (2013) Nicotinamide adenine dinucleotide-induced multimerization of the co-repressor CtBP1 relies on a switching tryptophan. J Biol Chem 288:27836-27848.

Mani-Telang P, Sutrias-Grau M, Williams G, and Arnosti DN (2007) Role of NAD binding and catalytic residues in the C-terminal binding protein corepressor. FEBS Lett 581:5241-5246.

McGaughey GB, Gagné M, and Rappé AK (1998) pi-Stacking interactions: alive and well in proteins. J Biol Chem 273:15458-15463.

Minard ME, Kim LS, Price JE, and Gallick GE (2004) The role of the guanine nucleotide exchange factor Tiam1 in cellular migration, invasion, adhesion and tumor progression. Breast Cancer Res Treat 84:21-32.

Morris BL (2016) Understanding and Targeting the C-Terminal Binding Protein (CtBP) Substrate-Binding Domain for Cancer Therapeutic Development. Doctoral dissertation, Virginia Commonwealth University, Richmond, VA.

Nardini M, Svergun D, Konarev PV, Spanò S, Fasano M, Bracco C, Pesce A, Donadini A, Cericola C, Secundo F, et al. (2006) The C-terminal domain of the transcriptional corepressor CtBP is intrinsically unstructured. Protein Sci 15:1042-1050.
Paliwal S, Ho N, Parker D, and Grossman SR (2012) CtBP2 promotes human cancer cell migration by transcriptional activation of Tiam1. Genes Cancer 3:481-490.

Quinlan KG, Nardini M, Verger A, Francescato P, Yaswen P, Corda D, Bolognesi M, and Crossley M (2006) Specific recognition of ZNF217 and other zinc finger proteins at a surface groove of C-terminal binding proteins. Mol Cell Biol 26: 8159-8172.

Schmittgen TD and Livak KJ (2008) Analyzing real-time PCR data by the comparative C(T) method. Nat Protoc 3:1101-1108.

Schuck P (2000) Size-distribution analysis of macromolecules by sedimentation velocity ultracentrifugation and lamm equation modeling. Biophys $J$ 78:1606-1619.

Shi Y, Sawada J, Sui G, Affar B, Whetstine JR, Lan F, Ogawa H, Luke MP, Nakatan $\mathrm{Y}$, and Shi Y (2003) Coordinated histone modifications mediated by a CtBP co-repressor complex. Nature 422:735-738.

Stankiewicz TR, Schroeder EK, Kelsey NA, Bouchard RJ, and Linseman DA (2013) C-terminal binding proteins are essential pro-survival factors that undergo caspase-dependent downregulation during neuronal apoptosis. Mol Cell Neurosci 56:322-332.

Straza MW, Paliwal S, Kovi RC, Rajeshkumar B, Trenh P, Parker D, Whalen GF, Lyle S, Schiffer CA, and Grossman SR (2010) Therapeutic targeting of C-terminal binding protein in human cancer. Cell Cycle 9:3740-3750.

Sumner ET, Chawla AT, Cororaton AD, Koblinski JE, Kovi RC, Love IM, Szomju BB Korwar S, Ellis KC, and Grossman SR (2017) Transforming activity and therapeutic targeting of C-terminal-binding protein 2 in Apc-mutated neoplasia. Oncogene 36:4810-4816.

Address correspondence to: Dr. Steven R. Grossman, Department of Internal Medicine, Virginia Commonwealth University, 1201 East Marshall Street, Box 980070, Richmond, VA 23298-0230. E-mail: steven.grossman@ vcuhealth.org 